\title{
Article \\ Hemifield-Specific Rotational Biases during the Observation of Ambiguous Human Silhouettes
}

\author{
Chiara Lucafò $*,+\mathbb{D}$, Daniele Marzoli ${ }^{*}+$, Caterina Padulo (D), Stefano Troiano, Lucia Pelosi Zazzerini, \\ Gianluca Malatesta (D), Ilaria Amodeo and Luca Tommasi
}

Department of Psychological Sciences, Health and Territory, University of Chieti, Via dei Vestini 29, I-66013 Chieti, Italy; caterina.padulo@unich.it (C.P.); stefano.troiano@studenti.unich.it (S.T.); lpelosi@libero.it (L.P.Z.); gianluca.malatesta@unich.it (G.M.); amodeoilaria90@gmail.com (I.A.); luca.tommasi@unich.it (L.T.)

* Correspondence: chiara.lucafo@unich.it (C.L.); d.marzoli@unich.it (D.M.)

+ These authors equally contributed to this work.

check for

updates

Citation: Lucafò, C.; Marzoli, D.;

Padulo, C.; Troiano, S.;

Pelosi Zazzerini, L.; Malatesta, G.;

Amodeo, I.; Tommasi, L.

Hemifield-Specific Rotational Biases during the Observation of

Ambiguous Human Silhouettes.

Symmetry 2021, 13, 1349. https:/ /

doi.org/10.3390/sym13081349

Academic Editor: Vilfredo De Pascalis

Received: 20 May 2021

Accepted: 26 June 2021

Published: 26 July 2021

Publisher's Note: MDPI stays neutral with regard to jurisdictional claims in published maps and institutional affiliations.

Copyright: (C) 2021 by the authors. Licensee MDPI, Basel, Switzerland. This article is an open access article distributed under the terms and conditions of the Creative Commons Attribution (CC BY) license (https:/ / creativecommons.org/licenses/by/ $4.0 /)$.

\begin{abstract}
Both static and dynamic ambiguous stimuli representing human bodies that perform unimanual or unipedal movements are usually interpreted as right-limbed rather than left-limbed, suggesting that human observers attend to the right side of others more than the left one. Moreover, such a bias is stronger when static human silhouettes are presented in the RVF (right visual field) than in the LVF (left visual field), which might represent a particular instance of embodiment. On the other hand, hemispheric-specific rotational biases, combined with the well-known bias to perceive forward-facing figures, could represent a confounding factor when accounting for such findings. Therefore, we investigated whether the lateralized presentation of an ambiguous rotating human body would affect its perceived handedness/footedness (implying a role of motor representations), its perceived spinning direction (implying a role of visual representations), or both. To this aim, we required participants to indicate the perceived spinning direction (which also unveils the perceived handedness/footedness) of ambiguous stimuli depicting humans with an arm or a leg outstretched. Results indicated that the lateralized presentation of the stimuli affected both their perceived limb laterality (a larger number of figures being interpreted as right-limbed in the RVF than in the LVF) and their perceived spinning direction (a larger number of figures being interpreted as spinning clockwise in the LVF than in the RVF). However, the hemifield of presentation showed a larger effect size on the perceived spinning direction than on the perceived limb laterality. Therefore, as we already proposed, the implicit representation of others' handedness seems to be affected more by visual than by motor processes during the perception of ambiguous human silhouettes.
\end{abstract}

Keywords: human body; ambiguous figures; handedness/footedness; divided visual field paradigm; lateralized embodiment; optic flow

\section{Introduction}

In recent studies [1-4], we found that when right- and left-handed subjects were required to indicate the orientation of ambiguous (in terms of front or back view) figures representing human silhouettes in the act of performing one-handed manual actions, both groups reported perceiving the figures more frequently as oriented consistently with a right-handed movement than with a left-handed movement. Similar findings were reported for dynamic stimuli representing ambiguous human bodies represented in the act of performing unimanual and unipedal movements $[3,5,6]$. These results indicate an attentional/perceptual bias toward the right side of the human body and are also consistent with findings from research investigating the perception of sport actions, according to which the outcomes of right-limbed movements can be anticipated better than the outcomes of leftlimbed movements [7-13]. Noteworthy, this difference is present regardless of observers' handedness $[7,11]$, which is in contrast with the proposal that the correspondence between 
observed movements and stored representations could foster action recognition (see [14] for a review). According to Hagemann [7] and Loffing, Schorer, et al. [9] (see also [8,10,11,13]), the ability to discriminate left-handed actions would be lower than that to discriminate right-handed actions (the same would hold true for foot movements), a difference likely due to a perceptual frequency effect (see also [15]): given that both right- and left-handers usually play against right- rather than left-handed opponents, the discrimination of righthanded actions would be easier for both groups in several interactive sports, in agreement with theories that emphasize the crucial role of visual experience in action perception (e.g., [16]). From an evolutionary point of view, an attentional and perceptual bias in favor of right-handed movements might be adaptive in social life because most everyday social interactions occur with right- rather than left-handed individuals (see also [17] for a more detailed discussion). Specifically, such a bias would represent a perceptual preference to attend to the body region most likely including others' right hand. Given that, compared with the left hand, the right hand is more used in both communicative and aggressive behaviors, the bias toward the right side of human bodies would increase the efficiency in monitoring such behaviors. Further findings in line with a bias toward the right limbs of human bodies are provided by studies employing biological motion represented through point-light animations [18]. For example, Thornton et al. [19] (see also [20]) found that a point-light walker exhibiting equal motion clues to either side, and hence ambiguous in terms of heading direction, was interpreted more often as right-facing than as left-facing. It should be noticed that, when a right-facing individual is observed, the right limbs are in the foreground, and thus such results support the presence of an attentional and perceptual bias in favor of the right side of others.

The preference for perceiving right-limbed actions did not correlate with the degree of participants' handedness in previous studies with ambiguous human body stimuli $[1-5,19,20]$, and thus it is plausible that such tasks involve relatively more visual than motor processes. Nonetheless, there is also some evidence for a role of motor representations, at least under certain conditions. Indeed, in a series of psychophysical experiments, de Lussanet et al. [21] clearly showed that rightward-facing point-light walkers were better identified compared with leftward-facing ones in the RVF (right visual field), whereas leftward-facing walkers were better identified compared with rightward-facing ones in the LVF (left visual field). To explain this lateralized facing effect, the authors proposed that the lateralized embodiment of an observed point-light walker is facilitated when the side of the visual cortex that processes the stimulus corresponds with the side of the sensory-motor cortices that process the hemibody seen in the foreground (for consistent findings with body part stimuli, see [22,23]). Moreover, we found that static human silhouettes with ambiguous handedness were interpreted more frequently as right-handed in the RVF than in the LVF [2,4]. Therefore, it is plausible that the hemifield of presentation promotes a laterally biased perception of ambiguous human bodies on the basis of the motor representations stored in the more stimulated (i.e., contralateral) hemisphere. However, there is some evidence suggesting that the hemifield of presentation might also affect the perceived spinning direction of ambiguous stimuli for other, non-motoric, reasons. In particular, as already proposed by [24], the usual experience of optic flow (i.e., the motion patterns of objects in the visual field of the observer; $[25,26])$ could induce hemispheric-specific rotational biases when one observes rotating objects with an ambiguous spinning direction. Indeed, the experience of optic flow (which occurs during walking, running, driving, and other activities implying a forward motion) is represented by the constant movement of objects "toward" the observer, but is also analogous to the perception of the motion vectors on the visible surface of a cylinder rotating CW (clockwise) in the LVF, and of those on the visible surface of a cylinder rotating CCW (counterclockwise) in the RVF. Specifically, Alipour and Kazemi [24] found a significantly shorter duration of the CCW perception when a stimulus (the spinning dancer designed by Japanese web artist Nobuyuki Kayahara; http:/ / www.procreo.jp/labo/labo13.html, accessed on 28 June 2021) was presented in the LVF rather than in the RVF. Moreover, Burton et al. [27] examined reaction times 
for CW and CCW mental rotations in the two hemifields, showing that CCW and CW rotations were more efficiently performed in the RVF and LVF, respectively. We point out that a hemifield-specific rotational bias could also account for previous findings with static human silhouettes [2-4]. Indeed, a perceptual bias for forward-facing motion exists [28,29] (see also [30]) that can also induce observers to interpret ambiguous figures consistently with a forward rather than backward motion [31-33] (see also [34]) and ambiguous human bodies as front-facing rather than back-facing [1-4,35,36] (for further instances of perceptual and attentional advantages for approaching rather than receding stimuli, see [37-41]). Therefore, the combination of the bias to perceive forward-facing figures (which would be consistent with an inward rather than outward rotation) and the aforementioned hemifieldspecific rotational bias could increase the proportion of left-sided silhouettes (i.e., those in which the action is depicted —-from the observer's point of view-on the figure's left side) and right-sided silhouettes (i.e., those in which the action is depicted on the figure's right side) interpreted as "potentially rotating" inward and CCW (and thus right-handed) in the RVF and as "potentially rotating" inward and CW (and thus left-handed) in the LVF. The same hemifield-specific rotational bias could account for de Lussanet et al.'s [21] findings: when a forward-facing stimulus located in one hemifield approaches (or is approached by) the observer, it appears to rotate until heading right (through a CCW rotation) or left (through a CW rotation) in the RVF and LVF, respectively. The same should apply to ambiguous stimuli (see [42]) because of the aforementioned perceptual bias for forward-facing motion. Interestingly, the hemifield-specific rotational bias might also account for certain links observed between perceptual and motor asymmetries (e.g., [43]). In summary, it has yet to be determined which specific factor is responsible for the tendency to interpret a larger number of ambiguous human bodies as right-handed in the RVF rather than in the LVF. Thus, the present study investigated whether the lateralized presentation of an ambiguous rotating human body affects its perceived handedness / footedness (in which case, mainly motor representations would be involved) or its perceived spinning direction (in which case, mainly visual representations would be involved), or both. To this aim, we used rotating stimuli depicting humans performing unimanual or unipedal movements.

\section{Materials and Methods}

In agreement with the recommendations by Simmons, Nelson, and Simonsohn [44], we report how we defined our sample sizes, as well as all manipulations, data exclusions, and measures in the study.

\subsection{Participants}

One hundred and twenty-eight subjects (64 females and 64 males; age: 18-39 years) participated in the study. Specifically, we scheduled the enrollment of eight male subjects and eight female subjects for each different combination of outstretched limb and response arrow spinning direction (CW or CCW), position (above or below), and color (red or green; see Procedure section). All subjects had normal or corrected-to-normal vision.

\subsection{Stimuli}

We used two sets of 128 animations obtained from an animation originally created with the software Poser Pro 2012 (Smith Micro Software Inc., Pittsburgh, PA, USA), which represented the silhouette of a human male rotating around his vertical axis while maintaining a static posture (in the first set of animations, the silhouette was depicted as standing on both legs with one arm outstretched and the other arm close to the body; in the second set of animations, the silhouette was depicted as standing on one leg with the other leg outstretched and both arms close to the body; Figure 1). Given that the silhouette is portrayed in black on a blank background and that no obvious depth clue is available, the animations turn out to be ambiguous and can be interpreted either as rotating CW (a percept consistent with an outstretched left arm or right leg in the original animations) or as rotating CCW (a percept consistent with an outstretched right arm or left leg in the 
original animations). Then, we decomposed each original animation into its 32 constituent frames and created 64 different versions, each of which depicted a complete rotation of the silhouette, by reorganizing the 32 frames on the basis of starting frame and order (i.e., from the 1st to the 32nd and vice versa, from the 2nd to the 1st and vice versa, and so on). Such an order manipulation permitted to offset any association between spinning direction and outstretched arm/leg by including two rotation conditions (e.g., see $[3,5,6])$. In the first condition, the silhouette turns inward (i.e., "palmward" with reference to the hand movement and "en dedans"- a term describing a spin in which the ballet dancer turns toward the supporting leg-with reference to the foot movement). In the second condition, the silhouette turns outward (i.e., "backward" with reference to the hand movement and "en dehors"- a term describing a spin in which the ballet dancer turns away from the supporting leg-with reference to the foot movement). In the "inward" order, the CW rotation is congruent with an outstretched left arm/leg, whereas in the "outward" order, the $\mathrm{CW}$ rotation is congruent with an outstretched right arm/leg, and vice versa for the $\mathrm{CCW}$ rotation. We set the Poser parameters (e.g., camera distance and camera elevation) so as to remove - to the extent possible-any potential perspective clue (e.g., relative size and relative height). However, we mirrored horizontally each frame so as to create a further set of 64 animations, which permitted to offset the effects of any remaining unwanted asymmetry or depth cue that might have biased the perception of the outstretched limb (obviously, this manipulation did not affect the association between spinning direction and outstretched arm/leg in either order condition/type of rotation; see examples of possible percepts at https:/ / osf.io/xrn4y /?view_only=cd558b56e03b4ff9a9c54eb16d464040, accessed on 28 June 2021). The 128 animations of each set of stimuli represented each possible combination of mirroring, type of rotation (inward or outward), and starting frame. Furthermore, these 128 final animations were presented once on the right side of the screen and once on the left side of the screen (after the onset of a fixation cross remaining in the center of the screen until stimulus offset) so as to obtain 256 trials. At a $57 \mathrm{~cm}$ viewing distance, the frames composing each animation measured about $10.3^{\circ}$ vertically and, on average, about $3.7^{\circ}$ horizontally. Half of the participants observed the first set of animations (silhouette with one arm outstretched) and the other half observed the second set of animations (silhouette with one leg outstretched).

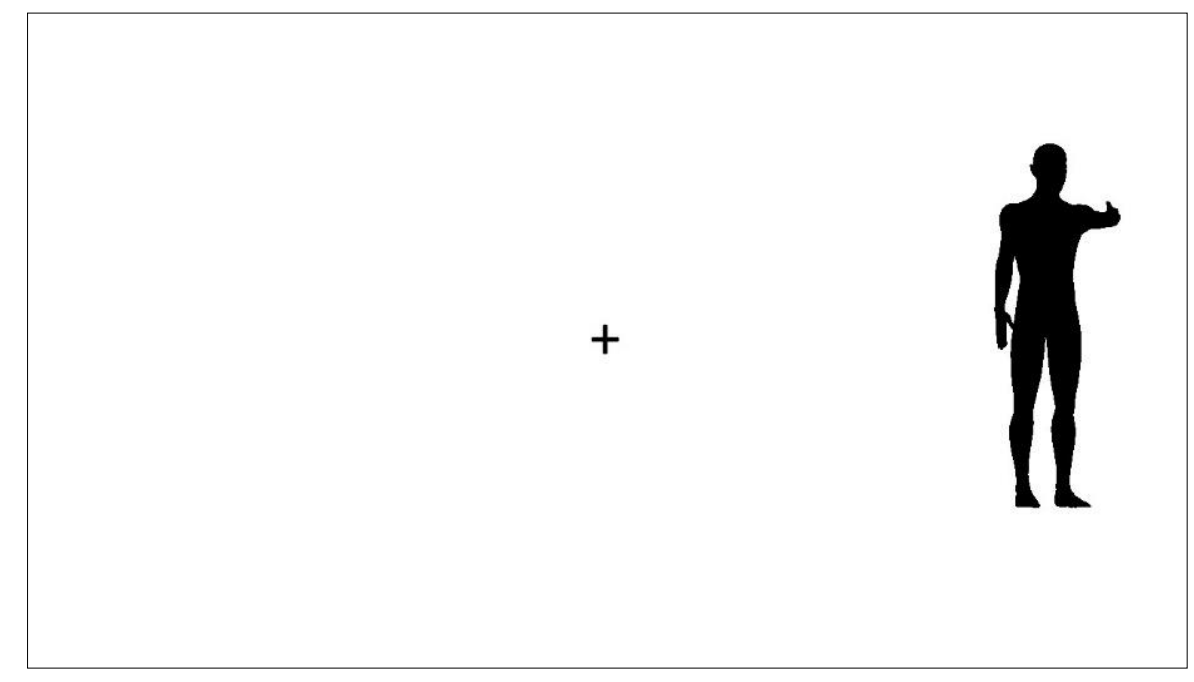

(a)

Figure 1. Cont. 


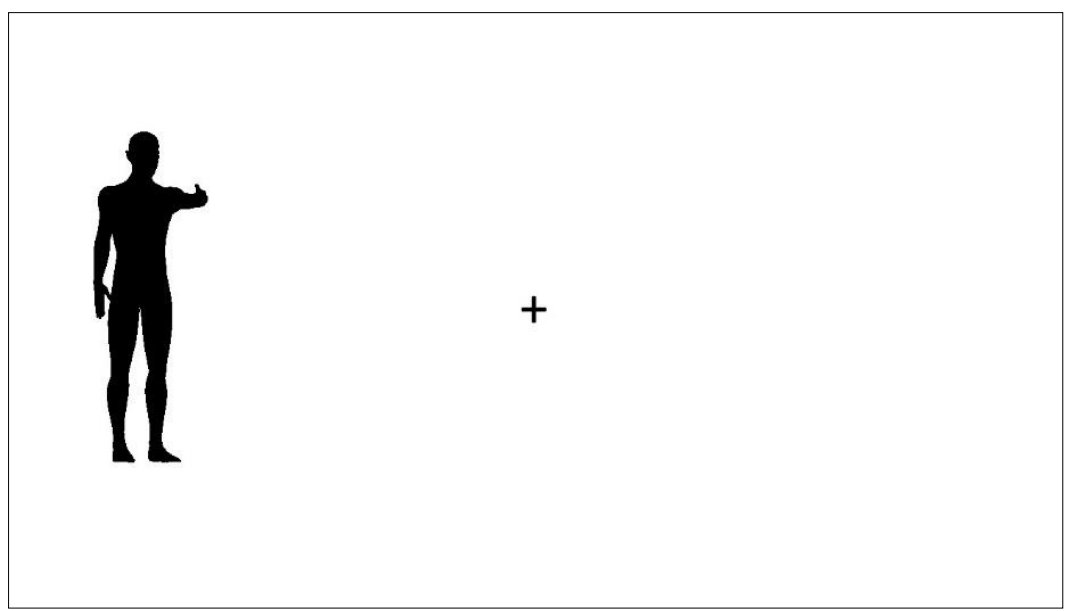

(b)

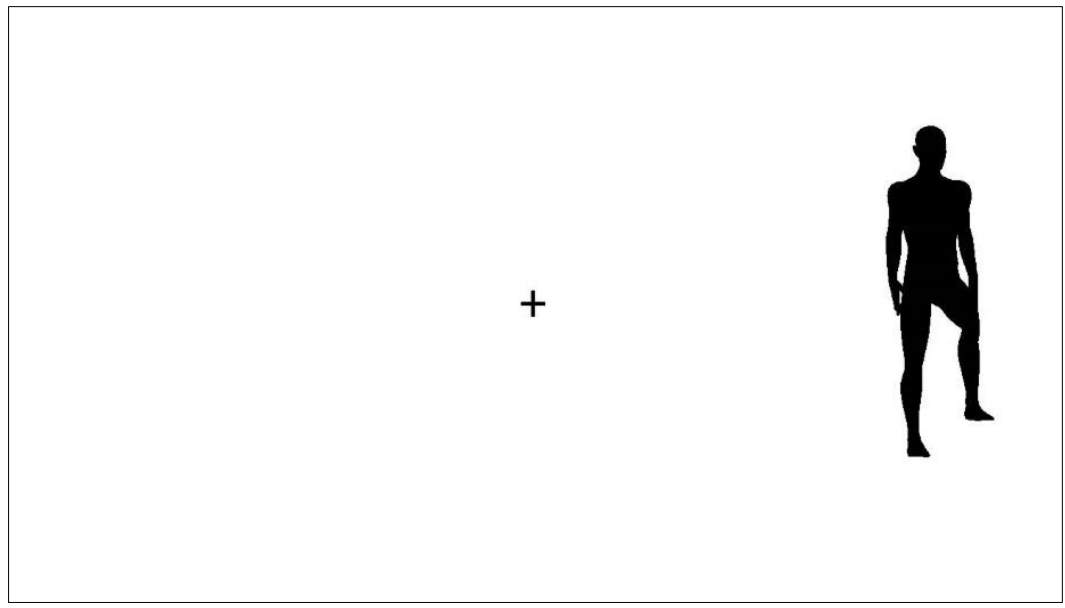

(c)

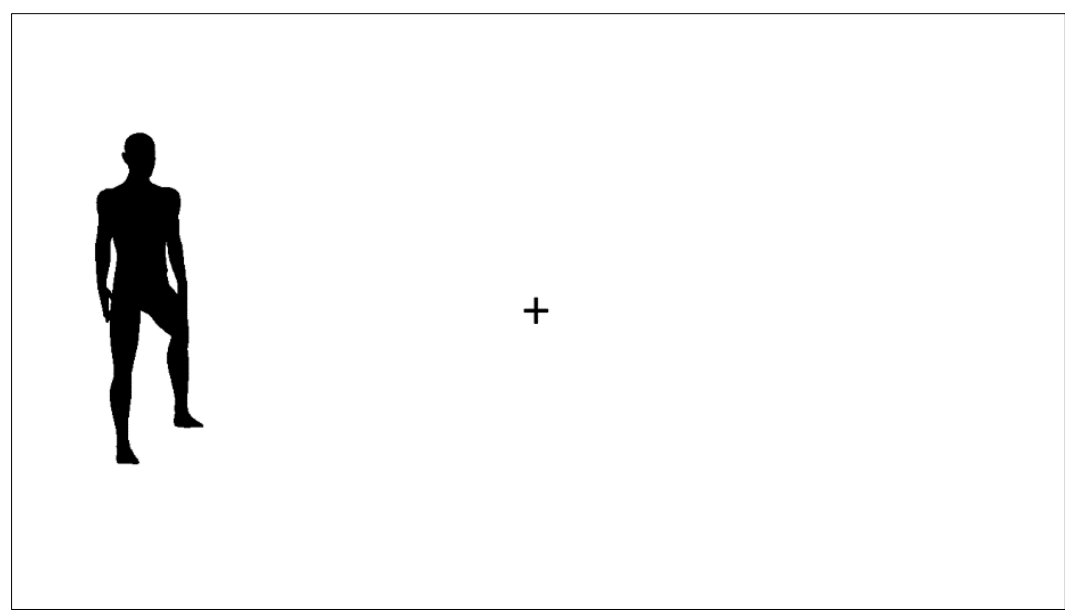

(d)

Figure 1. Examples of stimuli with an arm outstretched $(\mathbf{a}, \mathbf{b})$ and with a leg outstretched $(\mathbf{c}, \mathbf{d})$ on the right $(\mathbf{a}, \mathbf{c})$ and on the left $(\mathbf{b}, \mathbf{d})$ of the fixation cross.

\subsection{Procedure}

The experiment was performed with SuperLab 4.0 (Cedrus Corporation, San Pedro, CA, USA) on a Windows (Microsoft Corporation, Redmond, WA, USA) notebook equipped with an Intel (Intel Corporation, Santa Clara, CA, USA) processor and a 15.4-inch monitor. Participants were tested in a quiet room, seated at a $57 \mathrm{~cm}$ viewing distance from the 
computer screen, and they were invited to put their hands palms down on the table and not to cross any body part (i.e., legs, arms, or fingers) throughout the experiment. The experiment consisted of 256 trials (128 right trials and 128 left trials) in each of which a black fixation cross presented in the center of a blank screen was followed after $500 \mathrm{~ms}$ by one of the stimuli described previously presented laterally ( $12.4^{\circ}$ from the fixation point) for $2300 \mathrm{~ms}$ and then by a couple of colored arrows - one located slightly above and the other located slightly below the center of the screen-depicting the two possible spinning directions of the silhouette (Figure 2). Participants were asked to gaze at the fixation point and to report the perceived spinning direction of the silhouette by pronouncing the words "ROSSO" (the Italian word for "RED") and "VERDE" ("GREEN") according to their percept. After the participant's response was recorded by the experimenter, who was seated behind the participant and pressed the key " $\mathrm{R}$ " or " $\mathrm{V}$ " on a keyboard connected to the notebook, the next trial started.

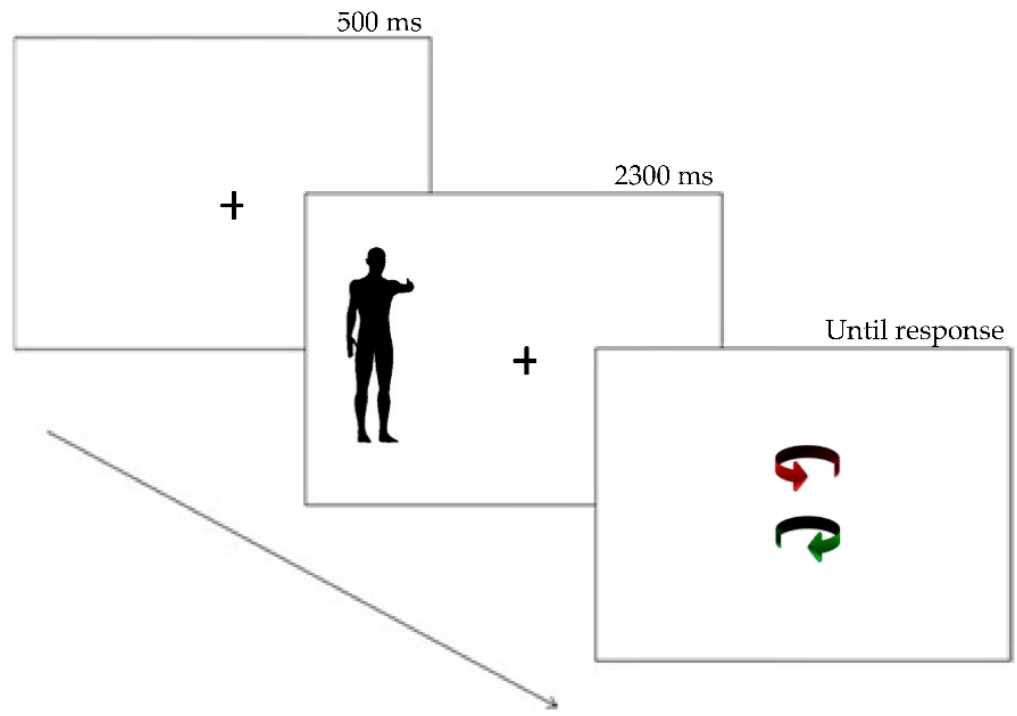

Figure 2. Schematic representation of the time course of a trial.

The first frame of each animation lasted $750 \mathrm{~ms}$, and the remaining 31 frames lasted $50 \mathrm{~ms}$. This expedient was adopted in order to reduce the possible carry-over of responses between consecutive trials (e.g., see [45], where percept carry-over was observed even when a $30 \mathrm{~s}$ break was introduced after the presentation of each ambiguous spinning dancer). Moreover, we collected subjects' responses by using two colored arrows, each depicting a possible spinning direction, rather than using simple vocal responses such as "ORARIO" and "ANTIORARIO" (the Italian words for "CLOCKWISE" and "COUNTERCLOCKWISE", respectively), given that the latter response modality turns out to be rather troublesome for observers (likely due to the difficulty in labeling as CW or CCW a rotation around an axis that is roughly parallel to one's own body axis). Before the experiment, participants were familiarized with the response modality by administering them a pretest in which they reported - by means of the two response arrows described above- the spinning direction of a black human silhouette that contained perspective cues (in particular, the relative size of the hands in distinct positions). Such a pretest permitted the exclusion from the study of the few subjects who were not able to perform the task. Each stimulus was shown twice, once to each hemifield, and the stimuli were presented in a random sequence.

The participant's face was recorded throughout the experiment by means of the computer-integrated webcam. This allowed the monitoring of her/his eye movements in order to discard trials in which she/he moved her/his gaze from the fixation point. Finally, the participant's hand preference was assessed by means of the Italian version of the Edinburgh Handedness Inventory [46]. The study was carried out following the 
guidelines of the Declaration of Helsinki, and it was authorized by the Institutional Review Board of Psychology of the Department of Psychological Sciences, Health, and Territory of the University of Chieti (protocol code 21003). A written informed consent was provided by each participant.

\subsection{Data Analysis}

Twenty-five subjects (15 males and 10 females) were excluded from the analysis due to problems in video recording, and 2 subjects (both male) were excluded because they always gave the same response (RED or GREEN). The trials in which subjects moved their eyes from the fixation point were eliminated, and 7 other subjects ( 4 males and 3 females) were excluded because the proportion of displacements exceeded $15 \%$. On the basis of their laterality score on the Italian version of the Edinburgh Handedness Inventory [46], the remaining 94 subjects were categorized as right-handers (87 subjects exhibiting a positive laterality score; range: $0.15 / 1.00 ; \mathrm{M}=0.65 \pm 0.023 \mathrm{SEM}$ ) or left-handers (7 subjects exhibiting a negative laterality score; range: $-0.81 /-0.14 ; \mathrm{M}=0.52 \pm 0.076 \mathrm{SEM}$ ).

Two different analyses were carried out to test whether (1) the perceived limb laterality and (2) the perceived spinning direction of the figure were affected by the hemifield of presentation, the type of rotation, the outstretched limb, participants' sex, and/or their interactions. Initially, we computed for each participant eight measures indicating the number of figures interpreted as right- and left-limbed and eight measures representing the number of figures interpreted as rotating CW and CCW in any combination of hemifield of presentation and type of rotation. Then, 2 female participants and 3 male participants were excluded as outliers because they scored at least 2 standard deviations above or below the average of their experimental group (arm or leg) in any of the aforementioned measures. Finally, we performed two repeated-measures analyses of variance (ANOVAs). In the first, we used participant's sex (female or male) and the outstretched limb (arm or leg) as between-subjects factors, and the side of hemifield presentation (right or left) and perceived limb laterality (right or left) as within-subjects factors. In the second, we used participant's sex (female or male) and the outstretched limb (arm or leg) as between-subjects factors, and the side of hemifield presentation (right or left) and perceived spinning direction (CW or $(C W)$ as within-subjects factors. As evident in Table 1, coding the percept according to limb laterality or spinning direction permutes the roles of main effects and interactions while leaving identical F- and $p$-values. When needed, we carried out post-hoc t-tests in order to determine the significant differences (within each set of post-hoc contrasts, $p$-values were adjusted according to the Bonferroni correction for multiple comparisons). Participants' handedness was not included as an independent variable in the ANOVAs performed due to the low number of left-handers, so participants' laterality score was correlated with the percentage of figures interpreted as right-limbed and the percentage of figures interpreted as rotating CW.

Table 1. Correspondences between the results of ANOVAs with percepts coded according to limb laterality and spinning direction. Effect size $\left(\eta_{\mathrm{p}}{ }^{2}\right)$ and observed power $(1-\beta)$ for each significant effect (computed for $\alpha=0.05$ ) are also reported.

\begin{tabular}{|c|c|c|}
\hline \multicolumn{2}{|c|}{ Factors } & \multirow{2}{*}{ Statistics } \\
\hline Perceived Limb Laterality & Perceived Spinning Direction & \\
\hline Perceived limb laterality & Type of rotation $X$ Perceived spinning direction & $\begin{array}{c}\mathrm{F}_{1,85}=3.994 \\
p=0.049 \\
\eta_{\mathrm{p}}^{2}=0.045 \\
1-\beta=0.506\end{array}$ \\
\hline $\begin{array}{l}\text { Hemifield of presentation X Perceived } \\
\text { limb laterality }\end{array}$ & $\begin{array}{l}\text { Hemifield of presentation } X \text { The type of rotation } X \\
\text { Perceived spinning direction }\end{array}$ & $\begin{array}{c}\mathrm{F}_{1,85}=5.376 \\
p=0.023 \\
\eta_{\mathrm{p}}^{2}=0.059 \\
1-\beta=0.630\end{array}$ \\
\hline
\end{tabular}


Table 1. Cont.

\begin{tabular}{|c|c|c|}
\hline \multicolumn{2}{|c|}{ Factors } & \multirow{2}{*}{ Statistics } \\
\hline Perceived Limb Laterality & Perceived Spinning Direction & \\
\hline Participant's sex X Perceived limb laterality & $\begin{array}{c}\text { Type of rotation X Participant's sex X Perceived } \\
\text { spinning direction }\end{array}$ & $\begin{array}{c}\mathrm{F}_{1,85}=7.763 \\
p=0.007 \\
\eta_{\mathrm{p}}^{2}=0.084 \\
1-\beta=0.787\end{array}$ \\
\hline $\begin{array}{l}\text { Hemifield of presentation } X \text { Type of rotation } X \\
\text { Perceived limb laterality }\end{array}$ & $\begin{array}{l}\text { Hemifield of presentation X Perceived } \\
\text { spinning direction }\end{array}$ & $\begin{array}{c}\mathrm{F}_{1,85}=11.654 \\
p<0.001 \\
\eta_{\mathrm{p}}{ }^{2}=0.121 \\
1-\beta=0.921\end{array}$ \\
\hline $\begin{array}{c}\text { Type of rotation X Outstretched limb X } \\
\text { Participant's sex X Perceived limb laterality }\end{array}$ & $\begin{array}{l}\text { Outstretched limb X Participant's sex X Perceived } \\
\text { spinning direction }\end{array}$ & $\begin{array}{c}\mathrm{F}_{1,85}=6.002 \\
p=0.016 \\
\eta_{\mathrm{p}}^{2}=0.066 \\
1-\beta=0.678\end{array}$ \\
\hline $\begin{array}{c}\text { Hemifield of presentation X Type of rotation X } \\
\text { Outstretched limb X Participant's sex X } \\
\text { Perceived limb laterality }\end{array}$ & $\begin{array}{l}\text { Hemifield of presentation X Outstretched limb X } \\
\text { Participant's sex X Perceived spinning direction }\end{array}$ & $\begin{array}{c}\mathrm{F}_{1,85}=4.576 \\
p=0.035 \\
\eta_{\mathrm{p}}^{2}=0.051 \\
1-\beta=0.562\end{array}$ \\
\hline
\end{tabular}

\section{Results}

The first ANOVA, which examined perceived limb laterality, showed: a significant main effect of perceived limb laterality $\left(\mathrm{F}_{1,85}=3.994 ; p=0.049\right)$; a significant interaction between the hemifield of presentation and perceived limb laterality $\left(\mathrm{F}_{1,85}=5.376 ; p=0.023\right)$; a significant interaction between participant's sex and perceived limb laterality $\left(\mathrm{F}_{1,85}=7.763\right.$; $p=0.007)$; a significant interaction between the hemifield of presentation, type of rotation, and perceived limb laterality $\left(\mathrm{F}_{1,85}=11.654 ; p<0.001\right)$; a significant interaction between the type of rotation, outstretched limb, participant's sex, and perceived limb laterality $\left(\mathrm{F}_{1,85}=6.002 ; p=0.016\right)$; and a significant interaction between the hemifield of presentation, type of rotation, outstretched limb, participant's sex, and perceived limb laterality $\left(\mathrm{F}_{1,85}=4.576 ; p=0.035\right)$. Participants' laterality score did not correlate with the percentage of figures interpreted as right-limbed $(\mathrm{n}=89 ; \mathrm{r}=-0.037 ; p=0.727)$.

Participants perceived a larger number of right-limbed $(\mathrm{M}=51.54 \%)$ rather than leftlimbed figures $(\mathrm{M}=48.46 \%)$. However, this difference held true in the RVF (right-limbed: $\mathrm{M}=52.54 \%$; left-limbed: $\mathrm{M}=47.46 \%$; $\mathrm{t}_{88}=2.675, p=0.018$ ) but not in the $\mathrm{LVF}$ (right-limbed: $\mathrm{M}=50.55 \%$; left-limbed: $\mathrm{M}=49.45 \% ; \mathrm{t}_{88}=0.558, p=1$ ). Moreover, a larger number of figures were interpreted as right-limbed in the RVF than in the LVF ( $\mathrm{t}_{88}=2.227, p=0.028$; Figure 3).

Male participants perceived a larger number of right- $(M=54.01 \%)$ rather than leftlimbed figures $\left(\mathrm{M}=45.99 \% ; \mathrm{t}_{39}=4.152 ; p<0.001\right)$, whereas no difference was observed in female participants (right-limbed: $\mathrm{M}=49.53 \%$; left-limbed: $\mathrm{M}=50.47 \% ; \mathrm{t}_{48}=-0.368$; $p=1$ ). Moreover, the number of figures interpreted as right-limbed was larger for male than for female participants $\left(\mathrm{t}_{87}=2.695 ; p=0.008\right.$; Figure 4$)$.

In the LVF, participants perceived a larger number of right- $(\mathrm{M}=58.08 \%)$ rather than left-limbed figures $\left(\mathrm{M}=41.92 \%\right.$; $\left.\mathrm{t}_{88}=3.164, p=0.009\right)$ in the outward-rotation condition and a larger number of left- $(M=56.99 \%)$ rather than right-limbed figures $(M=43.01 \%$; $\left.\mathrm{t}_{88}=2.613, p=0.042\right)$ in the inward-rotation condition. In the RVF, no difference was observed in both the outward- (right-limbed: $M=49.07 \%$; left-limbed: $M=50.93 \%$; $\mathrm{t}_{88}=-0.382, p=1$ ) and inward-rotation condition (right-limbed: $\mathrm{M}=56.01 \%$; left-limbed: $\mathrm{M}=43.99 \% ; \mathrm{t}_{88}=2.210, p=0.119$ ). Moreover, participants perceived a larger number of right-limbed figures in the RVF $(M=56.01 \%)$ than in the LVF $\left(M=43.01 \% ; t_{88}=3.490\right.$, $p=0.002)$ in the inward-rotation condition and a larger number of right-limbed figures in the LVF $(\mathrm{M}=58.08 \%)$ than in the RVF $\left(\mathrm{M}=49.07 \%\right.$; $\left.\mathrm{t}_{88}=2.678, p=0.018\right)$ in the outward-rotation condition. Finally, in the LVF participants perceived a larger number of 
right-limbed figures in the outward-rotation condition $(\mathrm{M}=58.08 \%)$ than in the inwardrotation condition $\left(\mathrm{M}=43.01 ; \mathrm{t}_{88}=3.108, p=0.005\right)$, whereas no difference was observed in the RVF (outward rotation: $M=49.07 \%$; inward rotation: $M=56.01 \%$; $\mathrm{t}_{88}=-1.443$, $p=0.305$; Figure 5).

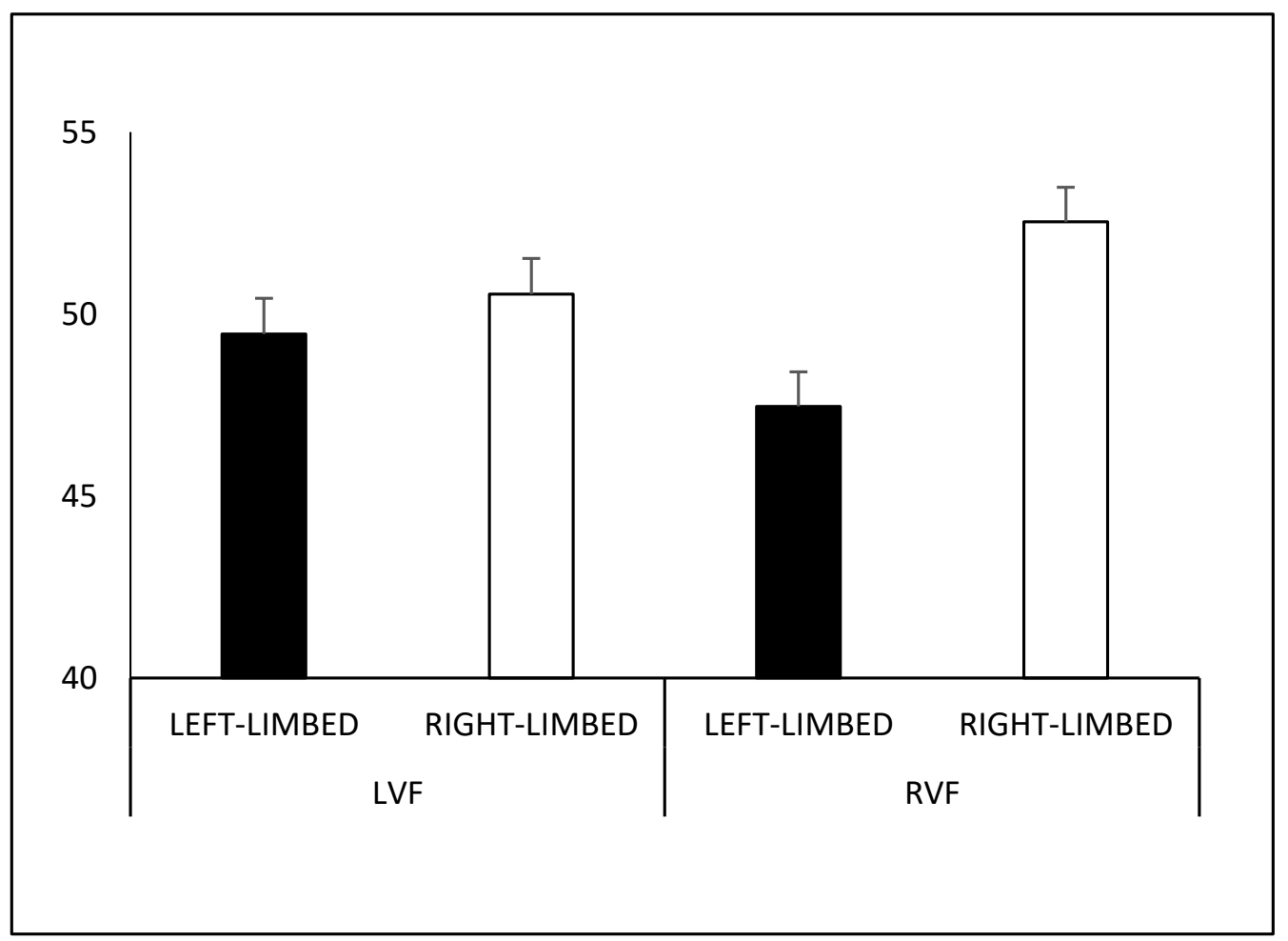

Figure 3. Percentage of figures interpreted as right- and left-limbed according to hemifield of presentation. Note: LVF, left visual field; RVF, right visual field.

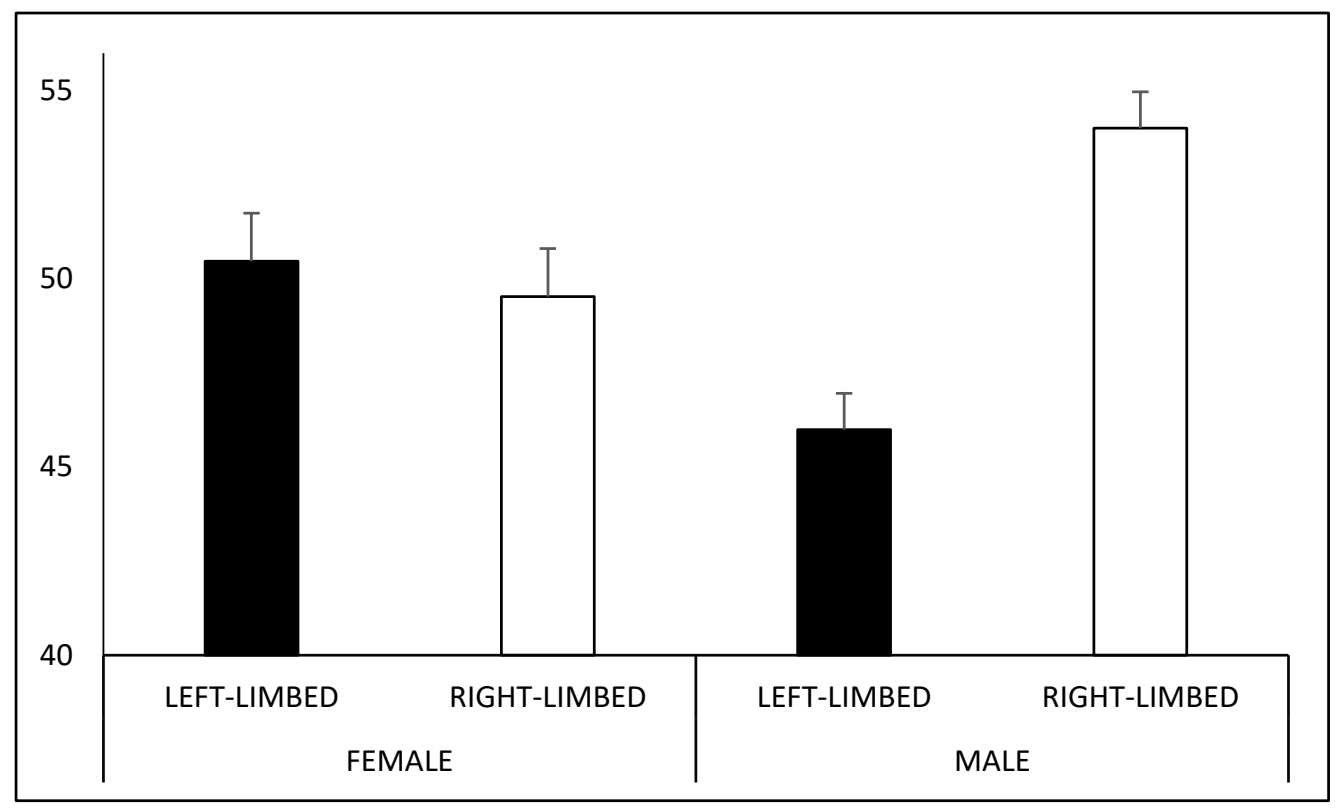

Figure 4. Percentage of figures interpreted as right- and left-limbed according to participant's sex. 


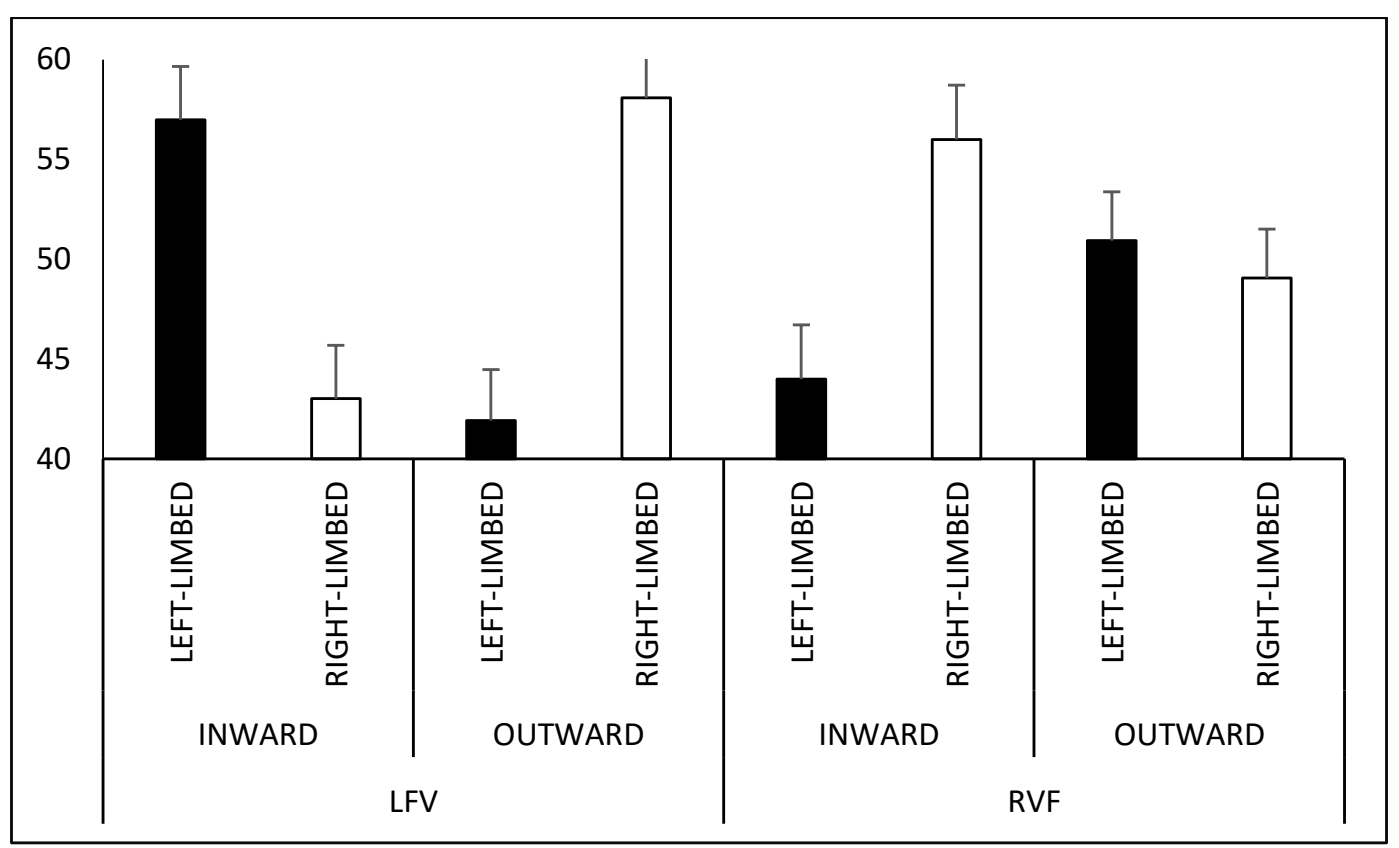

Figure 5. Percentage of figures interpreted as right- and left-limbed according to type of rotation and hemifield of presentation. Note: LVF, left visual field; RVF, right visual field.

In the inward-rotation condition of the leg-outstretched version of the experiment, male participants perceived a larger number of right-limbed figures $(\mathrm{M}=57.99 \%)$ compared with female participants $\left(\mathrm{M}=42.86 \% ; \mathrm{t}_{46}=3.060 ; p=0.015\right)$, and no significant difference was observed between male and female participants in the number of figures interpreted as right-limbed in any of the remaining combinations of outstretched limb and type of rotation (all $p>0.2$; Supplementary Material Table S1). Moreover, female participants showed a statistical trend to perceive a larger number of right-limbed figures in the outward-rotation condition of the leg-outstretched version of the experiment $(\mathrm{M}=58.47 \%)$ than in the outward-rotation condition of the arm-outstretched version of the experiment ( $\mathrm{M}=46.79 \%$; $\left.\mathrm{t}_{47}=2.497 ; p=0.064\right)$, and no significant difference was observed between the arm- and leg-outstretched versions of the experiment in the number of figures interpreted as rightlimbed in any of the remaining combinations of participant's sex and type of rotation (all $p>0.75$; Supplementary Material Table S2). Finally, female participants showed a statistical trend to perceive a larger number of right-limbed figures in the outward-rotation condition $(\mathrm{M}=58.47 \%)$ than in the inward-rotation condition ( $\left.\mathrm{M}=42.86 \% ; \mathrm{t}_{27}=2.616 ; p=0.058\right)$ of the leg-outstretched version of the experiment, and no significant difference was observed between the inward- and outward-rotation conditions in the number of figures interpreted as right-limbed in any of the remaining combinations of participant's sex and outstretched $\operatorname{limb}$ (all $p=1$; Supplementary Material Table S3).

In the inward-rotation condition of the leg-outstretched version of the experiment, male participants perceived a larger number of right- $(\mathrm{M}=69.06 \%)$ rather than left-limbed figures $\left(\mathrm{M}=30.94 \% ; \mathrm{t}_{19}=3.524 ; p=0.036\right)$ in the RVF, and no significant difference was observed between the number of left- and right-limbed figures in any of the remaining combinations of participant's sex, outstretched limb, type of rotation, and hemifield of presentation (all $p>0.3$; Supplementary Material Table S4). Moreover, in the leg-outstretched version of the experiment, male participants perceived a larger number of right-limbed figures in the RVF $(M=69.06 \%)$ than in the LVF $\left(M=46.92 \% ; t_{19}=3.299 ; p=0.030\right)$ in the inwardrotation condition and a larger number of right-limbed figures in the LVF $(\mathrm{M}=59.62 \%)$ than in the RVF $\left(M=40.53 \% ; \mathrm{t}_{19}=3.206 ; p=0.037\right)$ in the outward-rotation condition, and no significant difference was observed between the LVF and the RVF in the number of figures interpreted as right-limbed in any of the remaining combinations of participant's sex, outstretched limb, and type of rotation (all $p>0.08$; Supplementary Material Table S5). 
Finally, in the inward-rotation condition of the leg-outstretched version of the experiment, male participants perceived a larger number of right-limbed figures $(\mathrm{M}=69.06 \%)$ compared with female participants $\left(\mathrm{M}=44.71 \%\right.$; $\left.\mathrm{t}_{46}=3.121 ; p=0.025\right)$ in the $\mathrm{RVF}$, and no significant difference was observed between male and female participants in the number of figures interpreted as right-limbed in any of the remaining combinations of outstretched limb, type of rotation, and hemifield of presentation (all $p>0.08$; Supplementary Material Table S6).

The second ANOVA, which examined perceived spinning direction, showed: a significant interaction between the hemifield of presentation and perceived spinning direction $\left(\mathrm{F}_{1,85}=11.654 ; p<0.001\right)$; a significant interaction between the type of rotation and perceived spinning direction $\left(\mathrm{F}_{1,85}=3.994 ; p=0.049\right)$; a significant interaction between outstretched limb, participant's sex, and perceived spinning direction $\left(\mathrm{F}_{1,85}=6.002 ; p=0.016\right)$; a significant interaction between the type of rotation, participant's sex, and perceived spinning direction $\left(\mathrm{F}_{1,85}=7.763 ; p=0.007\right)$; a significant interaction between the hemifield of presentation, the type of rotation, and perceived spinning direction $\left(\mathrm{F}_{1,85}=5.376 ; p=0.023\right)$; and a significant interaction between the hemifield of presentation, outstretched limb, participant's sex, and perceived spinning direction $\left(\mathrm{F}_{1,85}=4.576 ; p=0.035\right)$. Participants laterality score did not correlate with the percentage of figures interpreted as spinning $\mathrm{CW}$ $(\mathrm{n}=89 ; \mathrm{r}=-0.094 ; p=0.382)$.

Participants perceived a larger number of CW- $(\mathrm{M}=57.53 \%)$ rather than CCWspinning figures $\left(\mathrm{M}=42.47 \% ; \mathrm{t}_{88}=3.108, p=0.005\right)$ in the LVF, whereas no difference was observed in the RVF (CW-spinning: $\mathrm{M}=46.53 \%$; CCW-spinning: $\mathrm{M}=53.47 \%$; $\mathrm{t}_{88}=-1.443$, $p=0.305)$. Moreover, a larger number of figures were interpreted as spinning $\mathrm{CW}$ in the LVF than in the RVF $\left(\mathrm{t}_{88}=3.204, p=0.002\right.$; Figure 6).

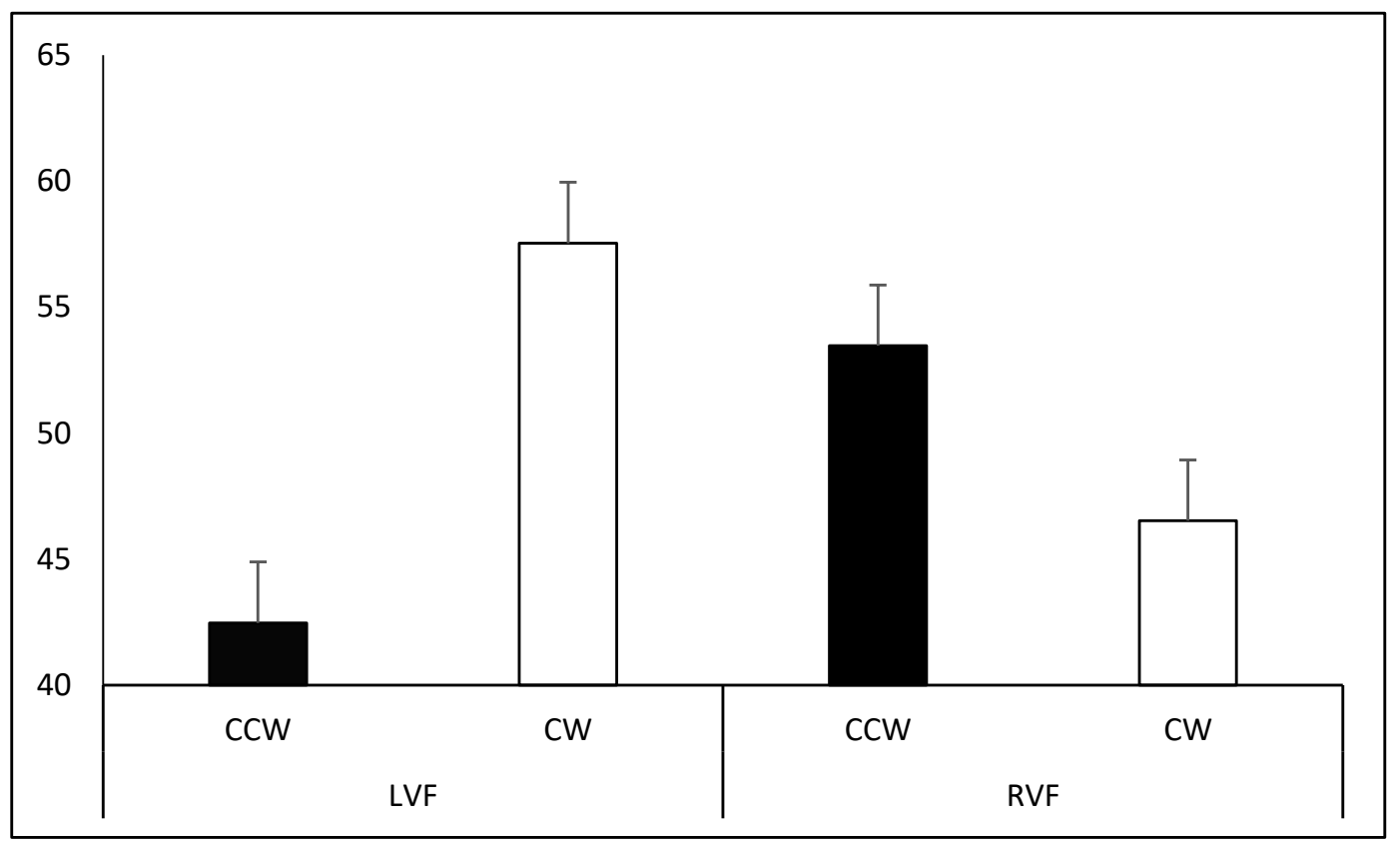

Figure 6. Percentage of figures interpreted as spinning clockwise and counterclockwise according to hemifield of presentation. Note: CW, clockwise; CCW, counterclockwise; LVF, left visual field; RVF, right visual field.

Participants showed a statistical trend to perceive a larger number of CW-spinning figures in the outward-rotation condition $(\mathrm{M}=53.57 \%)$ than in the inward-rotation condition $\left(\mathrm{M}=50.49 \% ; \mathrm{t}_{88}=1.803, p=0.075\right)$.

In the leg-outstretched version of the experiment, female participants perceived a larger number of $\mathrm{CW}$-spinning figures $(\mathrm{M}=57.80 \%)$ compared with male participants $\left(\mathrm{M}=46.04 \% ; \mathrm{t}_{46}=2.528 ; p=0.030\right)$, whereas no significant difference was observed between male and female participants in the number of figures interpreted as spinning $\mathrm{CW}$ in 
the arm-outstretched version of the experiment (female participants: $M=48.77 \%$; male participants: $\mathrm{M}=53.36 \% ; \mathrm{t}_{39}=0.965, p=0.681$ ). Moreover, female participants showed a statistical trend to perceive a larger number of $\mathrm{CW}(\mathrm{M}=57.80 \%)$ rather than $\mathrm{CCW}$-spinning figures $\left(M=42.20 \%\right.$; $\left.t_{27}=2.616, p=0.058\right)$ in the leg-outstretched version of the experiment, and no significant difference was observed between the number of figures interpreted as spinning CW and CCW in any of the remaining combinations of participant's sex and outstretched limb (all $p>1$; Supplementary Material Table S7).

Male participants perceived a larger number of CW-spinning figures in the outwardrotation condition $(\mathrm{M}=53.71 \%)$ than in the inward-rotation condition $(\mathrm{M}=45.70 \%$; $\left.\mathrm{t}_{39}=4.152, p<0.001\right)$, whereas no significant difference was observed in female participants (outward-rotation condition: $\mathrm{M}=53.46 \%$; inward-rotation condition: $\mathrm{M}=54.40 \%$; $\left.\mathrm{t}_{48}=-0.368, p=1\right)$. Moreover, in the inward-rotation condition, female participants showed a statistical trend to perceive a larger number of CW-spinning figures $(\mathrm{M}=54.40 \%)$ compared with male participants $\left(\mathrm{M}=45.70 \% ; \mathrm{t}_{87}=2.273, p=0.051\right.$; Figure 7$)$.

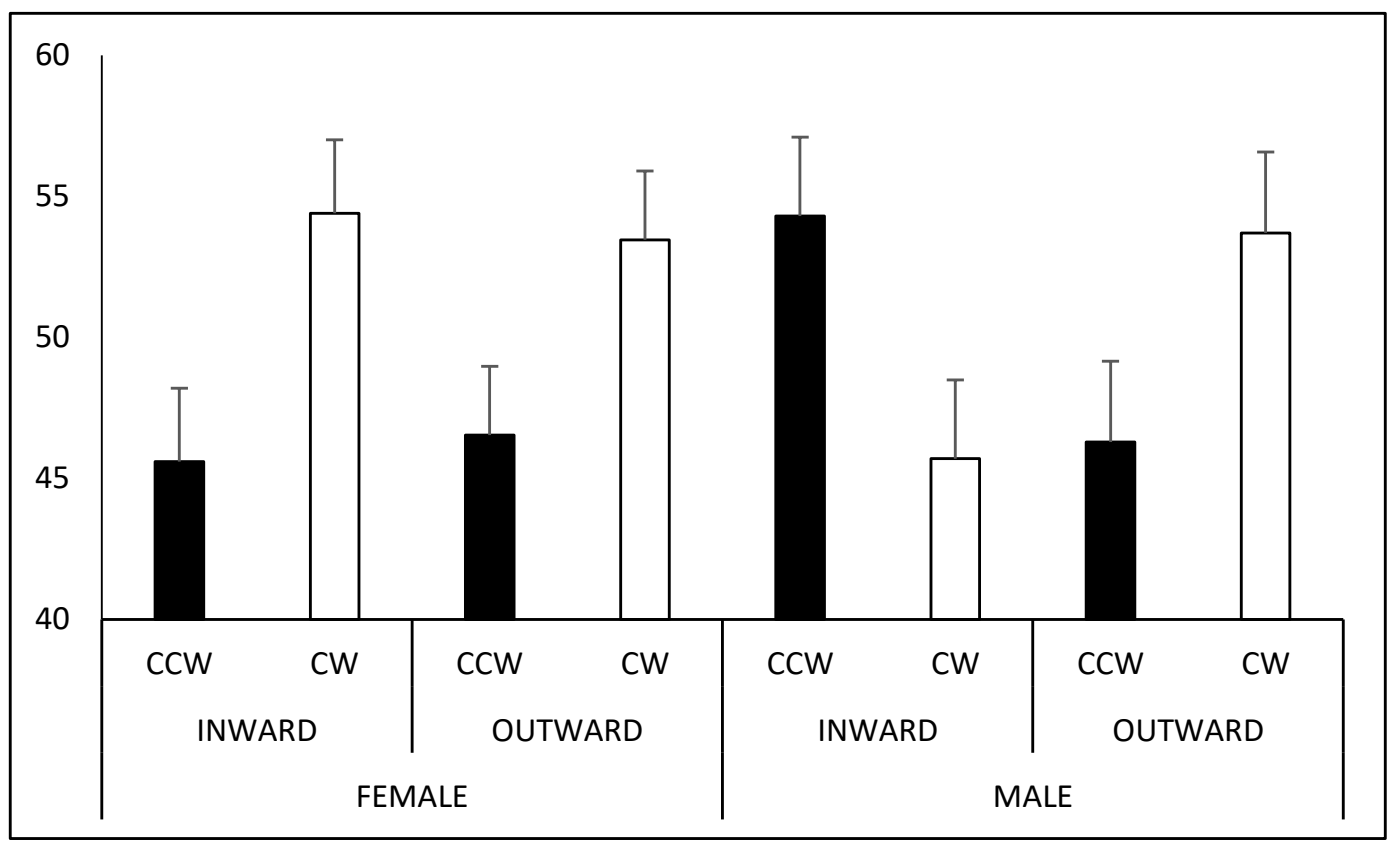

Figure 7. Percentage of figures interpreted as spinning clockwise and counterclockwise according to type of rotation and participant's sex. Note: CW, clockwise; CCW, counterclockwise.

In the LVF, participants perceived a larger number of CW- $(\mathrm{M}=56.99 \%)$ rather than CCW-spinning figures $\left(\mathrm{M}=43.01 \% ; \mathrm{t}_{88}=2.613, p=0.042\right)$ in the inward-rotation condition, and a larger number of $C W$-spinning figures $(\mathrm{M}=58.08 \%)$ rather than $\mathrm{CCW}$-spinning figures $\left(\mathrm{M}=41.92 \% ; \mathrm{t}_{88}=3.164, p=0.009\right)$ in the outward-rotation condition. In the RVF, no difference was observed in both the inward- (CW-spinning: $\mathrm{M}=43.99 \%$; $\mathrm{CCW}$-spinning: $\left.\mathrm{M}=56.01 \% ; \mathrm{t}_{88}=2.210, p=0.119\right)$ and the outward-rotation condition (CW-spinning: $\mathrm{M}=49.07 \%$; CCW-spinning: $\mathrm{M}=50.93 \% ; \mathrm{t}_{88}=-0.382, p=1$ ). Moreover, participants perceived a larger number of CW-spinning figures in the LVF (M =56.99\%) than in the RVF $\left(\mathrm{M}=43.99 \% ; \mathrm{t}_{88}=3.490, p=0.002\right)$ in the inward-rotation condition, and a larger number of CW-spinning figures in the LVF $(M=58.08 \%)$ than in the RVF $\left(M=49.07 \% ; t_{88}=2.678\right.$, $p=0.018$ ) in the outward-rotation condition. Finally, in the RVF, participants perceived a larger number of CW-spinning figures in the outward-rotation condition $(\mathrm{M}=49.07 \%)$ than in the inward-rotation condition $\left(\mathrm{M}=43.99 \%\right.$; $\left.\mathrm{t}_{88}=2.675, p=0.018\right)$, whereas no difference was observed in the LVF (outward-rotation: $M=58.08 \%$; inward-rotation: $M=56.99 \%$; $\mathrm{t}_{88}=0.558, p>1$; Figure 8). 


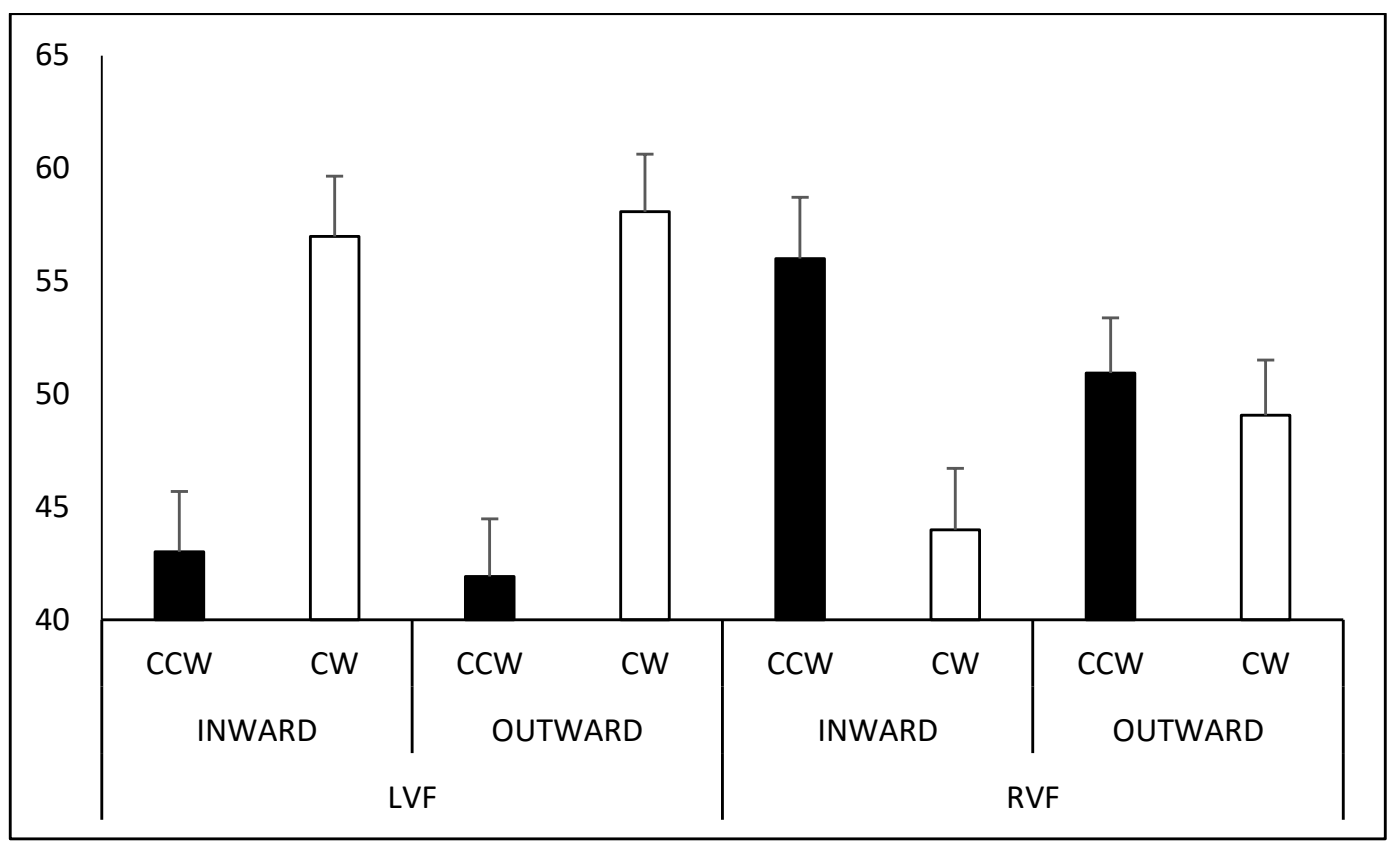

Figure 8. Percentage of figures interpreted as spinning clockwise and counterclockwise according to type of rotation and hemifield of presentation. Note: CW, clockwise; CCW, counterclockwise, LVF, left visual field; RVF, right visual field.

Male participants perceived a larger number of CW-spinning figures in the LVF $(\mathrm{M}=56.35 \%)$ than in the RFV (M = 35.73\%; $\left.\mathrm{t}_{19}=3.536, p=0.009\right)$ in the leg-outstretched version of the experiment but not in the arm-outstretched version of the experiment (CW-spinning in the LVF: $\mathrm{M}=56.19 \%$; $C W$-spinning in the RVF: $\mathrm{M}=50.54 \%$; $\mathrm{t}_{19}=0.911$, $p=1$ ), and female participants showed a statistical trend to perceive a larger number of CW-spinning figures in the LVF $(M=57.52 \%)$ than in the RFV $\left(M=40.03 \% ; \mathrm{t}_{20}=2.570\right.$, $p=0.073$ ) in the arm-outstretched version of the experiment but not in the leg-outstretched version of the experiment (CW-spinning in the LVF: $\mathrm{M}=59.35 \%$; $\mathrm{CW}$-spinning in the RVF: $\mathrm{M}=56.26 \% ; \mathrm{t}_{27}=0.424, p=1$ ). Moreover, in the leg-outstretched version of the experiment, female participants perceived a larger number of $\mathrm{CW}$-spinning figures $(\mathrm{M}=56.26 \%)$ compared with male participants $\left(\mathrm{M}=35.73 \%\right.$; $\left.\mathrm{t}_{46}=2.858, p=0.026\right)$ in the $\mathrm{RVF}$, and no difference was observed between male and female participants in the number of figures interpreted as spinning $\mathrm{CW}$ in any of the remaining combinations of hemifield of presentation and outstretched limb (all $p>0.22$; Supplementary Material Table S8). Finally, female participants showed a statistical trend to perceive a larger number of $\mathrm{CW}$-spinning figures in the leg-outstretched version of the experiment $(\mathrm{M}=56.26 \%)$ than in the arm-outstretched version of the experiment $\left(\mathrm{M}=40.03 \% ; \mathrm{t}_{47}=2.460, p=0.070\right)$ in the RVF, and no difference was observed between the arm- and leg-outstretched version of the experiment in the number of figures interpreted as spinning CW in any of the remaining combinations of hemifield of presentation and participant's sex (all $p>0.08$; Supplementary Material Table S9).

\section{Discussion}

In the present study, we investigated whether the lateralized presentation of rotating ambiguous human bodies affects their perceived handedness/footedness (in which case motor representations would be mainly involved) or their perceived spinning direction (in which case visual representations would be mainly involved), or even both. Results indicated that the lateralized presentation of the stimuli affected both their perceived limb laterality and their perceived spinning direction, although the effect size was larger in the latter $\left(\eta_{p}^{2}=0.06\right)$ than in the former $\left(\eta_{p}^{2}=0.12\right)$ case. In line with our previous studies with ambiguous human silhouettes [1-6], with the exception of one out of three experiments 
in [6], the participants' laterality score did not correlate with the percentage of figures interpreted as right-limbed or spinning CW.

As regards limb laterality, participants perceived a larger number of right-limbed rather than left-limbed figures. This effect corresponded to a statistical trend to interpret a larger number of figures as CW-spinning in the outward- rather than in the inward-rotation condition. These differences were significant in the RVF but not in the LVF, and a larger number of figures were interpreted as right-limbed in the RVF than in the LVF. These results are consistent with our previous studies showing that ambiguous human figures are interpreted more frequently as right- rather than left-limbed $[1-3,5,6]$ and that a larger number of such stimuli is interpreted as right-limbed in the RVF than in the LVF [4], indicating that the left and right hemispheres can prompt a biased interpretation of ambiguous stimuli, inducing the perception of right- and left-limbed movements, respectively. Therefore, the present study corroborates the proposal of a hemispheric specialization in the embodiment of observed movements, in line with previous studies with lateralized presentation of stimuli showing an association between the hemispheric representation of the observer's body and the visual representation of the observed bodies [21-23]. Moreover, male but not female participants perceived a larger number of right- rather than left-limbed figures (corresponding to the fact that only male participants interpreted a larger number of figures as CW-spinning in the outward-rather than in the inward-rotation condition), and the number of figures interpreted as right-limbed was larger for male than for female participants (corresponding to a statistical trend indicating that male participants, compared with female participants, interpreted a larger number of figures as CCW-spinning in the inwardrotation condition). This result might indicate that the bias for the right limb could be stronger in male rather than female individuals and seems to be consistent with the finding that the advantage of left-handers in sport is larger for male than for female players [47-49]. However, this conclusion must be considered with caution because the larger proportion of figures interpreted as right-limbed in male than in female participants was not predicted, and it was due mainly to the inward-rotation condition of the leg-outstretched version of the experiment (corresponding to the larger number of figures interpreted as spinning CCW by male than by female participants in the leg-outstretched version of the experiment) and more specifically to the inward-rotation condition of stimuli presented in the RVF in the leg-outstretched version of the experiment (corresponding to the larger number of figures presented in the RVF interpreted as spinning CCW by male than by female participants in the leg-outstretched version of the experiment). Thus, also because every significant effect including sex showed only a small to medium-low effect size (with $\eta_{\mathrm{p}}{ }^{2}$ ranging from 0.05 to 0.08 ) and because we observed inconsistent results in a previous study [6], this finding should be corroborated by further studies specifically aimed at testing the hypothesis that, body compared with females, males could exhibit a larger attentional and perceptual bias toward the right side of others.

As regards spinning direction, participants perceived a larger number of $\mathrm{CW}$ - rather than CCW-spinning figures in the LVF, whereas no difference was observed in the RVF. This effect corresponded to the fact that participants interpreted a larger number of figures as right-limbed in the outward- rather than in the inward-rotation condition in the LVF, whereas no difference was observed in the RVF. Moreover, a larger number of figures were interpreted as spinning CW in the LVF than in the RVF (corresponding to the fact that participants interpreted a larger number of figures as right-limbed in the RVF rather than in the LVF in the inward-rotation condition, and a larger number of figures as right-limbed in the LVF rather than in the RVF in the outward-rotation condition). These results are consistent with those reported by Alipour and Kazemi [24], who found analogous effects by presenting the spinning dancer illusion in the two hemifields. With the exception of that study, no previous research examined the effects of eccentricity on the perception of the spinning direction of bistable stimuli. As suggested by Alipour and Kazemi, it is plausible that the characteristics of optic flow, which can be represented by the motion vectors on the visible surfaces of two rotating cylinders (one located in the LVF and rotating 
$\mathrm{CW}$ and the other located in the RVF and rotating (CW) may help to explain this trend. In line with this interpretation, the study by Burton et al. [27] indicated that the right and left hemispheres show a bias for the CW and CCW direction, respectively. Such an optic-flow account may be criticized because it implicitly assumes a forward movement (i.e., approach) rather than a backward movement (i.e., recession) on behalf of either the observer or the stimulus. Nonetheless, it is important to emphasize how movements receding from the fixation point are much rarer than those approaching to it (humans, like other animals, usually move toward the objects they are looking at, and-from an evolutionary standpoint-fast backward movements constitute an exception also for humans, who experience it mainly when traveling by car, train, or ship). Furthermore, also as regards the stimuli used in this experiment, it is interesting to note how ambiguous stimuli representing humans (regardless of whether they consist of dynamic point-light displays or static silhouettes) are perceived more often as approaching/from the front than receding/from the back [1-4,35,36]. In particular, Schouten et al. [35] have suggested that an individual who is moving toward the observer should be a more relevant stimulus compared with an individual who is moving away. Accordingly, the visual system would assess the potential cost of misinterpreting others' actions and intentions, and assuming that someone is receding rather than approaching could be more costly compared with assuming that someone is approaching rather than receding (in particular, the facing bias would be congruent with the fact that approaching human walkers can convey a greater level of threat compared with receding human walkers). Therefore, also due to the absence of better alternative interpretations, the explanation based on the optic flow seems to be the most plausible (as well as in line with the different biological relevance of approaching compared with receding stimuli). As previously stated, the same hemifield-specific rotational bias could account for the tendency to perceive stimuli as moving away from the fixation point [21,42], a long since well-known perceptual preference that has often been linked to the common experience of optic flow during forward locomotion [50-55].

As already stated, the hemifield of presentation showed a larger effect size on the perceived spinning direction (with a medium-high $\eta_{p}{ }^{2}$ for the relevant interaction) than on the perceived limb laterality (with a medium $\eta_{p}{ }^{2}$ for the relevant interaction). These results corroborate our previous proposal that, differently from other tasks such as the imagination of others' actions [56-59], the implicit representation of others' handedness is affected more by visual than by motor processes during the perception of ambiguous human silhouettes [1-6]. Noteworthy, the same conclusion about a relatively greater role for motor and visual representations, respectively, during the imagination of others' actions [56-59] and the perception of ambiguous human silhouettes [1-6] can be drawn by the fact that a positive correlation between participants' right-handedness and the bias toward the right side of bodies is observed in the former but not in the latter task. The crucial role of perceptual processes is also corroborated by the finding that a specific training consisting in the visual presentation of right- and left-handedness can intensify or attenuate, respectively, the advantage in predicting the outcome of right- rather than lefthanded actions [13]. Another factor possibly affecting the bias toward the right limb might be stress, which is known to modulate hemispheric asymmetries, as well as cognitive and sport performance [60-63]. On the other hand, non-invasive brain stimulation has gained increasing popularity as a method to improve cognitive and sport performance [64-68], and one could wonder whether combining brain stimulation and perceptual training might exert even stronger effects in reducing the attentional and perceptual bias toward the right limb observed in sport.

The fact that the effect of the hemifield of presentation on the perceived spinning rotation overcame that on the perceived limb laterality could explain why in the present study the effect size for the main factor "perceived limb laterality" was small $\left(\eta_{\mathrm{p}}{ }^{2}=0.04\right)$ and relatively smaller compared with that observed in our previous studies using similar stimuli shown in central presentation, in which it ranged from medium $\left(\eta_{\mathrm{p}}{ }^{2}=0.10\right.$; unpublished data from [5]) to large $\left(\eta_{\mathrm{p}}^{2}=0.24 ;[6]\right)$. It should be also noticed that, differently from propos- 
als suggesting a role for embodiment in the hemifield-specific perceptual biases observed during the perception of human bodies or body parts [2,4,21-23], Williamson et al. [42] questioned the involvement of motor representations in biasing the perceived direction of point light-walkers in the two visual hemifields, pointing out that the bias to report perceiving right-facing walkers in the right visual field was not observed exclusively with stimuli representing human bodies. Aside from the differences in the experimental paradigm that necessarily limit the generalizability of such a conclusion, it should be considered with caution in light of our present findings, which show that when a possible confounding factor such as the perceptual bias for forward-facing motion is controlled for by using both inward- and outward-rotating stimuli, the hemifield of presentation biases both the perceived spinning direction and-although to a lesser extent—-the perceived limb laterality of ambiguous human bodies. Noteworthy, converging evidence seems to indicate, regardless of the stimuli used, a similar pattern of results: in central vision, an attentional and perceptual bias toward the right limb is observed when participants are required to report the orientation (front/back) of static ambiguous human silhouettes [1,3], the spinning direction (CW/CCW) of dynamic ambiguous human silhouettes $[3,5,6]$, and the facing direction (right/left) of masked point-light walkers [19,20]; in peripheral vision, as also observed in the present study, such a bias is abolished in the LVF and increased in the RVF [2,4,42]. A clearer picture of the role of visual and motor representations in lateral perceptual biases toward the human body could emerge from future studies investigating-in both central and peripheral vision-the correlations between the strength of such biases for static ambiguous human silhouettes, dynamic ambiguous human silhouettes, and masked point-light walkers. Further studies should also investigate the role of optic flow and related hemisphere-specific rotational biases in the relationship between motor and perceptual preferences (e.g., [43]; for reviews, see [69,70]).

Finally, it is worth addressing some possible limitations of the present study. First, we asked participants to report the perceived spinning direction of the silhouette by indicating which of two colored arrows represented their percept. Whereas such a response modality undoubtedly unveils whether participants perceived a CW or CCW rotation, the association between the perceived spinning direction and the perceived handedness of the silhouette is obviously less straightforward, although reasonable. In order to legitimate our decision, we would like to point out that observers can report more easily the perceived spinning direction rather than the perceived laterality of ambiguous stimuli like those adopted here, and nonetheless - as also found in the present study - a population bias is observed as regards perceived limb laterality but not perceived spinning direction $[3,5,6]$. Therefore, it is plausible that - at least on an implicit level — the perceived spinning direction of the silhouette unveils its perceived limb laterality, an idea that would be strongly corroborated if a positive correlation were found between the perceptual bias for the right limb observed in studies with ambiguous human silhouettes [1-6] and the perceptual advantage for right-limbed movements observed in sport studies [7-13]. Second, we discarded trials in which participants moved their gaze from the fixation point through a visual inspection of the video recordings of their face, acquired during the experiment, rather than by using an eye-tracking device, which might reduce the precision of our exclusion procedure. This limit could be overcome by resorting to eye-tracking measures in future studies.

Supplementary Materials: The following are available online at https: / www.mdpi.com/article / 10.3390/sym13081349/s1, File S1: Supplementary Tables S1-S9. Table S1: Percentage of figures perceived as right-limbed by female and male participants according to outstretched limb and type of rotation; Table S2: Percentage of figures perceived as right-limbed in the arm- and leg-outstretched versions of the experiment according to participant's sex and type of rotation; Table S3: Percentage of figures perceived as right-limbed in the inward- and outward-rotation conditions according to participant's sex and type of rotation; Table S4: Percentage of figures perceived as right- and left-limbed according to participant's sex, outstretched limb, hemifield of presentation and type of rotation Table S5: Percentage of figures perceived as right-limbed in each hemifield according to participant's sex, outstretched limb and type of rotation; Table S6: Percentage of figures perceived as right-limbed 
by female and male participants according to outstretched limb, hemifield of presentation and type of rotation; Table S7: Percentage of figures perceived as spinning clockwise (CW) and counterclockwise (CCV) according to participant's sex and outstretched limb; Table S8: Percentage of figures perceived as spinning clockwise $(\mathrm{CW})$ by female and male participants according to outstretched limb and hemifield of presentation; Table S9: Percentage of figures perceived as spinning clockwise (CW) in the arm- and leg-outstretched versions of the experiment according to participant's sex and hemifield of presentation.

Author Contributions: Conceptualization, C.L. and D.M.; methodology, C.L. and D.M.; formal analysis, C.L., D.M., C.P., S.T. and G.M.; investigation, C.L., C.P., S.T., L.P.Z. and I.A.; resources, L.T.; writing-original draft preparation, C.L. and D.M.; writing-review and editing, C.P., S.T., L.P.Z., G.M., I.A. and L.T.; visualization, C.L. and G.M.; supervision, D.M. and L.T.; project administration, D.M. All authors have read and agreed to the published version of the manuscript.

Funding: This research received no external funding.

Institutional Review Board Statement: The study was conducted according to the guidelines of the Declaration of Helsinki, and approved by the Institutional Review Board of Psychology of the Department of Psychological Sciences, Health, and Territory of the University of Chieti (protocol code 21003).

Informed Consent Statement: Informed consent was obtained from all subjects involved in the study.

Data Availability Statement: The data that support the findings of this study are available from the corresponding authors upon request.

Conflicts of Interest: The authors declare no conflict of interest.

\section{References}

1. Marzoli, D.; Lucafò, C.; Pagliara, A.; Cappuccio, R.; Brancucci, A.; Tommasi, L. Both Right- and Left-Handers Show a Bias to Attend Others' Right Arm. Exp. Brain Res. 2015, 233, 415-424. [CrossRef]

2. Marzoli, D.; Pagliara, A.; Prete, G.; Malatesta, G.; Lucafò, C.; Padulo, C.; Brancucci, A.; Tommasi, L. Lateralized Embodiment of Ambiguous Human Silhouettes: Data on Sex Differences. Data Brief 2019, 25, 104009. [CrossRef] [PubMed]

3. Marzoli, D.; Lucafò, C.; Padulo, C.; Prete, G.; Giacinto, L.; Tommasi, L. Inversion Reveals Perceptual Asymmetries in the Configural Processing of Human Body. Front. Behav. Neurosci. 2017, 11, 126. [CrossRef] [PubMed]

4. Marzoli, D.; Pagliara, A.; Prete, G.; Malatesta, G.; Lucafò, C.; Padulo, C.; Brancucci, A.; Tommasi, L. Hemispheric Asymmetries in the Processing of Body Sides: A Study with Ambiguous Human Silhouettes. Neurosci. Lett. 2017, 656, 114-119. [CrossRef]

5. Lucafò, C.; Marzoli, D.; Prete, G.; Tommasi, L. Laterality Effects in the Spinning Dancer Illusion: The Viewing-from-above Bias Is Only Part of the Story. Br. J. Psychol. Lond. Engl. 2016, 107, 698-709. [CrossRef] [PubMed]

6. Lucafò, C.; Marzoli, D.; Zdybek, P.; Malatesta, G.; Smerilli, F.; Ferrara, C.; Tommasi, L. The Bias toward the Right Side of Others Is Stronger for Hands than for Feet. Symmetry 2021, 13, 146. [CrossRef]

7. Hagemann, N. The Advantage of Being Left-Handed in Interactive Sports. Atten. Percept. Psychophys. 2009, 71, 1641-1648. [CrossRef]

8. Loffing, F.; Hagemann, N.; Schorer, J.; Baker, J. Skilled Players' and Novices' Difficulty Anticipating Left- vs. Right-Handed Opponents' Action Intentions Varies across Different Points in Time. Hum. Mov. Sci. 2015, 40, 410-421. [CrossRef]

9. Loffing, F.; Schorer, J.; Hagemann, N.; Baker, J. On the Advantage of Being Left-Handed in Volleyball: Further Evidence of the Specificity of Skilled Visual Perception. Atten. Percept. Psychophys. 2012, 74, 446-453. [CrossRef]

10. Loffing, F.; Hagemann, N. Zum Einfluss Des Anlaufwinkels Und Der Füßigkeit Des Schützen Auf Die Antizipation von Elfmeterschüssen. Z. Sportpsychol. 2015. [CrossRef]

11. Loffing, F.; Hagemann, N. Motor Competence Is Not Enough: Handedness Does Not Facilitate Visual Anticipation of SameHanded Action Outcome. Cortex 2020, 130, 94-99. [CrossRef] [PubMed]

12. McMorris, T.; Colenso, S. Anticipation of Professional Soccer Goalkeepers When Facing Right-and Left-Footed Penalty Kicks. Percept. Mot. Skills 1996, 82, 931-934. [CrossRef]

13. Schorer, J.; Loffing, F.; Hagemann, N.; Baker, J. Human Handedness in Interactive Situations: Negative Perceptual Frequency Effects Can Be Reversed! J. Sports Sci. 2012, 30, 507-513. [CrossRef]

14. Knoblich, G.; Flach, R. Action Identity: Evidence from Self-Recognition, Prediction, and Coordination. Conscious. Cogn. 2003, 12, 620-632. [CrossRef]

15. Faurie, C.; Raymond, M. Handedness, Homicide and Negative Frequency-Dependent Selection. Proc. Biol. Sci. 2005, 272, 25-28. [CrossRef]

16. Jacobs, A.; Pinto, J.; Shiffrar, M. Experience, Context, and the Visual Perception of Human Movement. J. Exp. Psychol. Hum. Percept. Perform. 2004, 30, 822-835. [CrossRef] [PubMed] 
17. Marzoli, D.; Prete, G.; Tommasi, L. Perceptual Asymmetries and Handedness: A Neglected Link? Front. Psychol. 2014, 5, 163. [CrossRef]

18. Johansson, G. Visual Perception of Biological Motion and a Model for Its Analysis. Percept. Psychophys. 1973, 14, $201-211$. [CrossRef]

19. Thornton, I.M.; Vuong, Q.C.; Bülthoff, H.H. A Chimeric Point-Light Walker. Perception 2003, 32, 377-383. [CrossRef] [PubMed]

20. Williamson, K.E.; Jakobson, L.S.; Troje, N.F. A Right-Facing Bias in the Processing of Biological Motion? J. Vis. 2008, 8, 913. [CrossRef]

21. de Lussanet, M.H.E.; Fadiga, L.; Michels, L.; Seitz, R.J.; Kleiser, R.; Lappe, M. Interaction of Visual Hemifield and Body View in Biological Motion Perception. Eur. J. Neurosci. 2008, 27, 514-522. [CrossRef] [PubMed]

22. Aziz-Zadeh, L.; Iacoboni, M.; Zaidel, E. Hemispheric Sensitivity to Body Stimuli in Simple Reaction Time. Exp. Brain Res. 2006, 170, 116-121. [CrossRef]

23. Parsons, L.M.; Gabrieli, J.D.; Phelps, E.A.; Gazzaniga, M.S. Cerebrally Lateralized Mental Representations of Hand Shape and Movement. J. Neurosci. Off. J. Soc. Neurosci. 1998, 18, 6539-6548. [CrossRef]

24. Alipour, A.; Kazemi, S. Dominant Right Hemisphere Controls Biased Rotation Perception. Psychol. Neurosci. $2015,8,435$. [CrossRef]

25. Burton, A.; Radford, J. Thinking in Perspective: Critical Essays in the Study of Thought Processes; Routledge: Abingdon, UK, 1978; Volume 646.

26. Horn, B.; Klaus, B.; Horn, P. Robot Vision; MIT Press: Cambridge, MA, USA, 1986.

27. Burton, L.A.; Wagner, N.; Lim, C.; Levy, J. Visual Field Differences for Clockwise and Counterclockwise Mental Rotation. Brain Cogn. 1992, 18, 192-207. [CrossRef]

28. McBeath, M.K.; Morikawa, K. Forward-Facing Motion Biases for Rigid and Nonrigid Biologically Likely Transformations. Percept. Mot. Skills 1997, 85, 1187-1193. [CrossRef]

29. McBeath, M.K.; Morikawa, K.; Kaiser, M.K. Perceptual Bias for Forward-Facing Motion. Psychol. Sci. 1992, 3, 362-367. [CrossRef]

30. Hsu, P.; Taylor, J.E.T.; Pratt, J. Frogs Jump Forward: Semantic Knowledge Influences the Perception of Element Motion in the Ternus Display. Perception 2015, 44, 779-789. [CrossRef]

31. Bernstein, L.J.; Cooper, L.A. Direction of Motion Influences Perceptual Identification of Ambiguous Figures. J. Exp. Psychol. Hum. Percept. Perform. 1997, 23, 721-737. [CrossRef]

32. Pavlova, M.; Krägeloh-Mann, I.; Birbaumer, N.; Sokolov, A. Biological Motion Shown Backwards: The Apparent-Facing Effect. Perception 2002, 31, 435-443. [CrossRef]

33. Wotton, J.M.; Ferragamo, A.C. Ambiguous Animals: Mutable Mammal Biases Bird. Perception 2012, 41, 501-503. [CrossRef]

34. Bucher, N.M.; Palmer, S.E. Effects of Motion on Perceived Pointing of Ambiguous Triangles. Percept. Psychophys. 1985, 38, 227-236. [CrossRef]

35. Schouten, B.; Troje, N.F.; Brooks, A.; van der Zwan, R.; Verfaillie, K. The Facing Bias in Biological Motion Perception: Effects of Stimulus Gender and Observer Sex. Atten. Percept. Psychophys. 2010, 72, 1256-1260. [CrossRef]

36. Vanrie, J.; Dekeyser, M.; Verfaillie, K. Bistability and Biasing Effects in the Perception of Ambiguous Point-Light Walkers. Perception 2004, 33, 547-560. [CrossRef]

37. Doi, H.; Shinohara, K. Bodily Movement of Approach Is Detected Faster than That of Receding. Psychon. Bull. Rev. 2012, 19, 858-863. [CrossRef]

38. Franconeri, S.L.; Simons, D.J. Moving and Looming Stimuli Capture Attention. Percept. Psychophys. 2003, 65, 999-1010. [CrossRef]

39. Lewis, C.F.; McBeath, M.K. Bias to Experience Approaching Motion in a Three-Dimensional Virtual Environment. Perception 2004, 33, 259-276. [CrossRef]

40. Parker, A.; Alais, D. A Bias for Looming Stimuli to Predominate in Binocular Rivalry. Vision Res. 2007, 47, 2661-2674. [CrossRef] [PubMed]

41. Skarratt, P.A.; Cole, G.G.; Gellatly, A.R.H. Prioritization of Looming and Receding Objects: Equal Slopes, Different Intercepts. Atten. Percept. Psychophys. 2009, 71, 964-970. [CrossRef] [PubMed]

42. Williamson, K.; Jakobson, L.; Troje, N. Life Detection in Central and Peripheral Vision. J. Vis. 2007, 7, 484. [CrossRef]

43. Malatesta, G.; Marzoli, D.; Tommasi, L. Keep a Left Profile, Baby! The Left-Cradling Bias Is Associated with a Preference for Left-Facing Profiles of Human Babies. Symmetry 2020, 12, 911. [CrossRef]

44. Simmons, J.P.; Nelson, L.D.; Simonsohn, U. A 21 Word Solution; Social Science Research Network: Rochester, NY, USA, 2012.

45. Liu, C.-H.; Tzeng, O.J.L.; Hung, D.L.; Tseng, P.; Juan, C.-H. Investigation of Bistable Perception with the "Silhouette Spinner": Sit Still, Spin the Dancer with Your Will. Vis. Res. 2012, 60, 34-39. [CrossRef] [PubMed]

46. Salmaso, D.; Longoni, A.M. Problems in the Assessment of Hand Preference. Cortex J. Devoted Study Nerv. Syst. Behav. 1985, 21, 533-549. [CrossRef]

47. Breznik, K. On the Gender Effects of Handedness in Professional Tennis. J. Sports Sci. Med. 2013, 12, 346-353. [PubMed]

48. Loffing, F.; Hagemann, N.; Strauss, B. Left-Handedness in Professional and Amateur Tennis. PLoS ONE 2012, 7, e49325. [CrossRef]

49. Raymond, M.; Pontier, D.; Dufour, A.B.; Møller, A.P. Frequency-Dependent Maintenance of Left Handedness in Humans. Proc. Biol. Sci. 1996, 263, 1627-1633. [CrossRef] [PubMed]

50. Annett, M. Individual Variation in Directional Bias in Visual Perception. Perception 1983, 12, 71-84. [CrossRef]

51. Ball, K.; Sekuler, R. Human Vision Favors Centrifugal Motion. Perception 1980, 9, 317-325. [CrossRef] 
52. Dumoulin, S.O.; Baker, C.L.; Hess, R.F. Centrifugal Bias for Second-Order but Not First-Order Motion. JOSA A 2001, 18, 2179-2189. [CrossRef]

53. Fahle, M.; Wehrhahn, C. Motion Perception in the Peripheral Visual Field. Graefes Arch. Clin. Exp. Ophthalmol. 1991, 229, 430-436. [CrossRef] [PubMed]

54. Georgeson, M.A.; Harris, M.G. Apparent Foveofugal Drift of Counterphase Gratings. Perception 1978, 7, 527-536. [CrossRef] [PubMed]

55. Lappe, M.; Rauschecker, J.P. Motion Anisotropies and Heading Detection. Biol. Cybern. 1995, 72, 261-277. [CrossRef] [PubMed]

56. Marzoli, D.; Menditto, S.; Lucafò, C.; Tommasi, L. Imagining Others' Handedness: Visual and Motor Processes in the Attribution of the Dominant Hand to an Imagined Agent. Exp. Brain Res. 2013, 229, 37-46. [CrossRef] [PubMed]

57. Marzoli, D.; Lucafò, C.; Rescigno, C.; Mussini, E.; Padulo, C.; Prete, G.; D’Anselmo, A.; Malatesta, G.; Tommasi, L. Sex-Specific Effects of Posture on the Attribution of Handedness to an Imagined Agent. Exp. Brain Res. 2017, 235, 1163-1171. [CrossRef]

58. Marzoli, D.; Mitaritonna, A.; Moretto, F.; Carluccio, P.; Tommasi, L. The Handedness of Imagined Bodies in Action and the Role of Perspective Taking. Brain Cogn. 2011, 75, 51-59. [CrossRef]

59. Marzoli, D.; Palumbo, R.; Di Domenico, A.; Penolazzi, B.; Garganese, P.; Tommasi, L. The Relation between Self-Reported Empathy and Motor Identification with Imagined Agents. PLoS ONE 2011, 6, e14595. [CrossRef]

60. Berretz, G.; Packheiser, J.; Wolf, O.T.; Ocklenburg, S. Dichotic Listening Performance and Interhemispheric Integration after Stress Exposure. Sci. Rep. 2020, 10, 20804. [CrossRef]

61. Schultheiss, O.C.; Riebel, K.; Jones, N.M. Activity Inhibition: A Predictor of Lateralized Brain Function during Stress? Neuropsychology 2009, 23, 392-404. [CrossRef]

62. Hardy, L. Psychological Stress, Performance, and Injury in Sport. Br. Med. Bull. 1992, 48, 615-629. [CrossRef]

63. Palmer, L. The Relationship Between Stress, Fatigue, and Cognitive Functioning. Coll. Stud. J. 2013, 47, 312-325.

64. Penolazzi, B.; Di Domenico, A.; Marzoli, D.; Mammarella, N.; Fairfield, B.; Franciotti, R.; Brancucci, A.; Tommasi, L. Effects of Transcranial Direct Current Stimulation on Episodic Memory Related to Emotional Visual Stimuli. PLoS ONE 2010, 5. [CrossRef]

65. Friehs, M.A.; Dechant, M.; Vedress, S.; Frings, C.; Mandryk, R.L. Shocking Advantage! Improving Digital Game Performance Using Non-Invasive Brain Stimulation. Int. J. Hum.-Comput. Stud. 2021, 148, 102582. [CrossRef]

66. Martin, D.M.; Liu, R.; Alonzo, A.; Green, M.; Loo, C.K. Use of Transcranial Direct Current Stimulation (TDCS) to Enhance Cognitive Training: Effect of Timing of Stimulation. Exp. Brain Res. 2014, 232, 3345-3351. [CrossRef] [PubMed]

67. Ruf, S.P.; Fallgatter, A.J.; Plewnia, C. Augmentation of Working Memory Training by Transcranial Direct Current Stimulation (TDCS). Sci. Rep. 2017, 7, 876. [CrossRef] [PubMed]

68. Friehs, M.A.; Güldenpenning, I.; Frings, C.; Weigelt, M. Electrify Your Game! Anodal TDCS Increases the Resistance to Head Fakes in Basketball. J. Cogn. Enhanc. 2020, 4, 62-70. [CrossRef]

69. Karim, A.K.M.R.; Proulx, M.J.; Likova, L.T. Anticlockwise or Clockwise? A Dynamic Perception-Action-Laterality Model for Directionality Bias in Visuospatial Functioning. Neurosci. Biobehav. Rev. 2016, 68, 669-693. [CrossRef]

70. Rogers, L.J. Asymmetry of Motor Behavior and Sensory Perception: Which Comes First? Symmetry 2020, 12, 690. [CrossRef] 